\title{
Microlenses for stereoscopic image formation
}

\author{
R. P. Rocha, J. P. Carmo, and J. H. Correia \\ Dept. Industrial Electronics, University of Minho, Campus Azurem, 4800-058 Guimaraes, Portugal \\ Email: rrocha@dei.uminho.pt, phone/fax: +351-253510-190/+351-253510-189
}

\begin{abstract}
This paper presents microlenses for integration on a stereoscopic image sensor in CMOS technology for use in biomedical devices. It is intended to provide an image sensor with a stereoscopic vision. An array of microlenses potentiates stereoscopic vision and maximizes the color fidelity. An array of optical filters tuned at the primary colors will enable a multicolor usage. The material selected for fabricating the microlens was the AZ4562 positive photoresist. The reflow method applied to the photoresist allowing the fabrication of microlenses with high reproducibility.
\end{abstract}

Keywords-Microlenses, optical filters, RGB, image sensor, stereoscopic vision, Low-cost fabrication.

\section{INTRODUCTION}

Currently, the available image sensing technology is not yet ready for stereoscopic acquisition. The final quality of the image will be improved because of the stereoscopic vision but also due to the system's high resolution. Typically, two cameras are used to achieve a two points of view (POV) perspective effect. But this solution presents some problems mainly because the two POVs, being sufficiently different, cause the induction of psycho-visual confusion by the user [1]. The parallax effect (see Figure 1) is used to achieve stereoscopic vision.
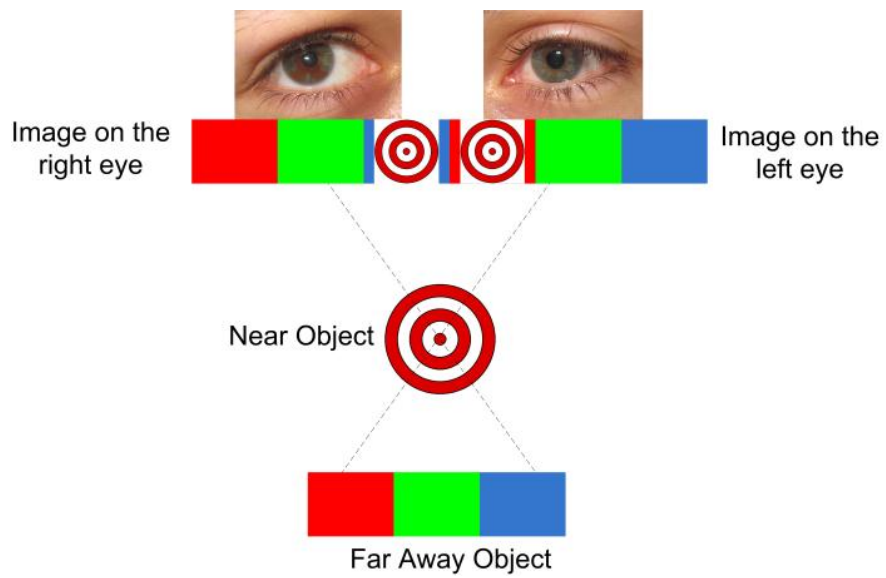

Figure 1: Illustration of the parallax effect for inducing sensation of depth in the human brain [1].

The result is obtained by "tricking" the brain causing it to gain depth perception (also known as stereopsis). This means that bad quality stereoscopy induces perceptual ambiguity in the viewer [2]. The reason for this phenomenon is that the human brain is simultaneously more sensitive but less tolerant to corrupt stereo images as well as vertical shifts of both images, being more tolerant to monoscopic images. Therefore, the brain does not consent the differences between the images coming from the left and right channels that are originated from the two independent and optically unadjusted cameras. A very interesting application for this type of imaging technology is in the biomedical field. Wireless capsule endoscopy, that take pictures along the digestive system, present themselves as excellent devices where to apply these image sensors [3,4], broadening the possibilities of noninvasive examination methods. Furthermore, the outspread of low-power small dimensioned high-resolution cameras motivates even more the fabrication of the next generation of endoscopic swallowable pills. These capsules can be electronically controlled for several purposes, from guidance inside the digestive system to drug delivery mechanisms. Presently, the capsules determine where they are according to the $\mathrm{pH}$ levels in their surroundings based on the different acidity profiles across the digestive system. Another advantage of the proposed imaging sensor is the compatibility with the CMOS technology. Most notably, CMOS allows the decrease of the power consumption and, unlike the CCD technology, CMOS sensors can be fabricated using the same manufacturing facilities as the high-volume products of the semiconductor industry. Thus, CMOS microdevices are more standardized and cost-effective to produce then the CCDs microdevices. Considering the low-power consumption of the CMOS microdevices, another possibility that presents itself for the imaging sensors is the integration of thin-films batteries with silicon compatibility [5] and low toxicity [6]. The inherent characteristics of the proposed imaging sensor presented in this paper make it an interesting solution for the capsule endoscopy integration including the thin-films battery as the power source. The fabrication of such a microdevice can be done using the $0.7 \mu \mathrm{m}$ CMOS process in the AMIS foundry because it allows the production of both the photodetectors and the read-out electronics within a reasonable cost. Finally, since the aforementioned process is very well characterized and known, the time to manufacture the first working prototype is expected to be short. This paper addresses on the design and fabrication technology of the microlenses for providing stereoscopic vision.

\section{IMAGE SENSOR ARCHITECTURE}

The image sensor is composed by two pupils (two entrance apertures just like the human eyes) from where the left and right channels (the two POVs that will originate the tridimensional effect) pass through before being focused by an objective lens. This lens focuses the two incident beams in the direction of the microlens, where the light is concentrated in a small sensitive area where the photodiodes are placed. After passing through the optical filters, the wavelengths coming from both the entrance apertures are directed towards the respective CMOS photodiodes. The two viewpoints are 
separated by focusing each side onto the appropriate sensor column under the microlens and optical filters.

A glass substrate is coated with a layer of $A Z 4562$ and exposed to a pattern with a mask

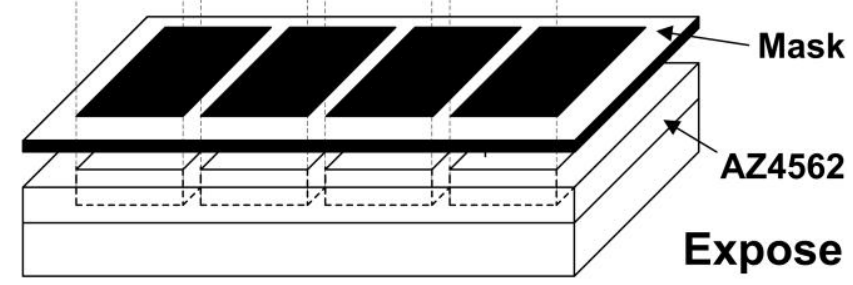

After the exposure, the AZ4562 is developed

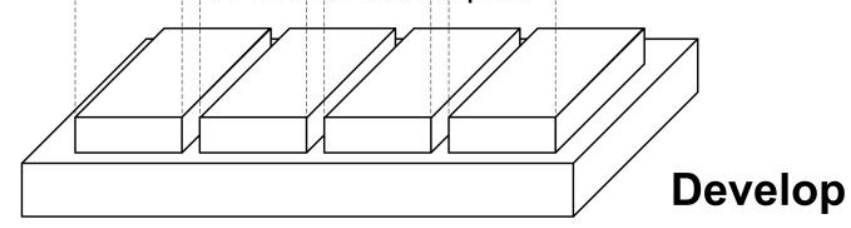

After being heated, the AZ4562 becomes viscous and forms a parabolic-shaped surface due to the surface tension

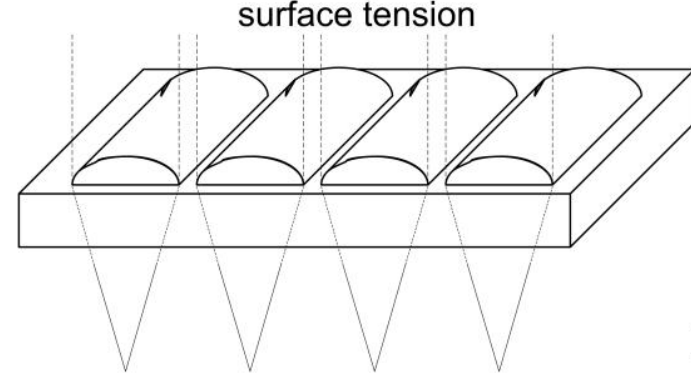

Reflow

Figure 2: The reflow process applied to the AZ4562 photoresist for fabricating microlenses arrays.

\section{MICROLENSES FABRICATION}

\section{A. Microlens fabrication}

There is a huge number of materials available for fabricating microlenses arrays such as the SU-8/2, AZ9260 and AZ4562, for example. These polymers allow the microlens fabrication by thermally reflowing the raw material, whose processing steps of the reflow process are presented in the Figure 2. This permits the production of arrays containing a million or more microlens of good optical quality in just a few minutes and with high degree of reproducibility of their characteristics. There are a few geometrical parameters to take in account before fabricating the microlenses. As depicted in Figure 3, the paraxial focal length, f, of a microlens consisting of a single spherical surface of radius $R$ in a medium of refractive index, $n$, is given by $f=R /(n-1)$ and the height, $h$, of the surface undulation of a microlens with an aperture radius, $r$, is $h=R-\left(R^{2}-r^{2}\right)^{1 / 2}$. The volume of a cylinder of resist is $T \pi r^{2}$, where $\mathrm{T}$ is the thickness before melting and the volume of the microlens is $1 / 3 \pi \mathrm{h}^{2}(3 \mathrm{R}-\mathrm{h})$. It therefore follows that the necessary thickness is given by $\mathrm{T}=\mathrm{h} / 6 \times\left[3+(\mathrm{h} / \mathrm{r})^{2}\right]$. The reflow process works well for microlenses of a relatively high-numerical aperture (and low-focal length, f), where the lens constitutes a significant fraction of a hemisphere. This process is very tolerant of the state of resist before melting, thus, acceptable lenses can be made with aperture ratios between $\mathrm{f} / 1$ and $\mathrm{f} / 3$.

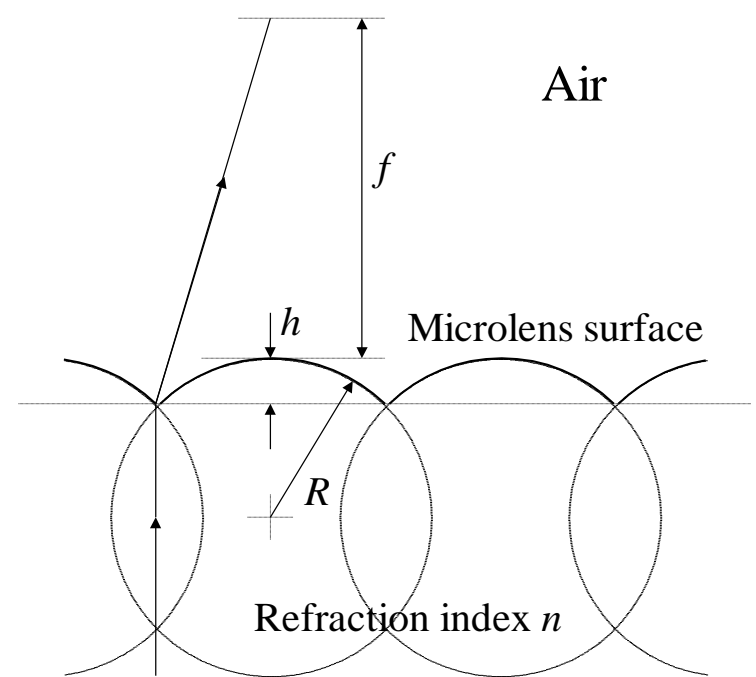

Figure 3: Factors determining the focal length of microlens.

Good-quality lenses are fabricated with relatively crude lithography although greater consistency and reliability is achieved only if the process is carried out efficiently. The precise form of the microlenses, and hence their focal properties, are determined by the effects of the surface tension [7]. In particular the contact angle of the softened resist with the surface of the substrate will strongly influence the shape of the microlens.

\section{B. Microlens simulation}

In Figure 4 it is possible to see the results of some finite element method (FEM) simulations. These simulations were performed considering the impinging light with an angle of $7.6^{\circ}$ degrees from the left channel, but this methodology is also applicable to the right channel and both channels overlapping for the global effect. The dimensions of the presented microlenses simulations have a W/L ratio of 4.8 and 2.4 in (a) and (b), respectively. It is clearly seen that the light concentrates in the direction of the photodiodes represented as pairs of black rectangles on the bottom of the figure. The simulations also show that the best results, i.e. more concentration of light, are achieved with microlenses having a higher curvature. Moreover, another important conclusion is shown, that it is possible to separate the left and right channels for focusing the specific wavelengths into the respective photodiodes. This also allows estimating the cross-talk between adjacent photodiodes, being smaller in the micro lenses with higher curvature. It is assumed in the simulations that the light had already passed through the optical filters. 


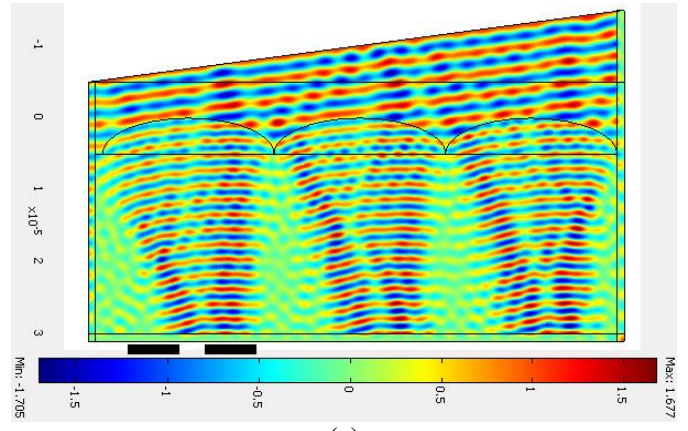

(a)

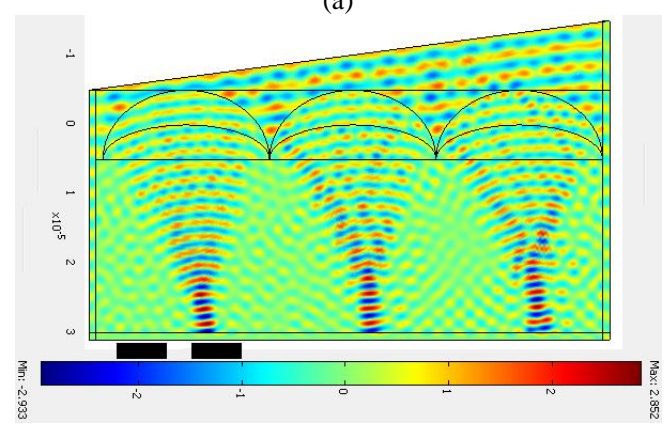

(b)

Figure 4: FEM simulations showing the light concentration into the right photodiode. The simulations where obtained respectively for lenses measuring with (a) $W / L=4.8$ and (b) $W / L=2.4$. The simulations also allows to roughly estimate the degree of cross talk between two adjacent photodiodes (e.g., between the left and right channels).

\section{EXPERIMENTAL}

\section{A. Fabrication}

The fabrication process of the microlenses array requires several steps. First, it is necessary to deposit the photoresist by means of a spin coater in a previously cleaned substrate. To achieve the desired thickness, the spin velocity is $6000 \mathrm{rpm}$ and the time will depend on the desired thickness. After the coating, a prebake phase, using a computer controlled hot plate at $100^{\circ} \mathrm{C}$ for 100 seconds, is necessary to evaporate the solvents present in the photoresist. This step is critical otherwise the resist surface dries very fast and doesn't allow the solvent to exit the photoresist creating bubbles and possibly the photoresist to lift. Next, to obtain the required array-like structure, the mask with the correspondent geometrical design is placed on top of the substrate with the photoresist. This step serves for exposing the PR to UV light, during 2 minutes, to make the unexposed material insoluble. Afterwards, the development phase, using the AZ 351B developer in a 1:4 concentration with distilled water, is required to leave just the unexposed photoresist in the substrate. To do this, the substrate is immersed in the developer bath in a magnetic stirrer for 1 minute. Finally, the structure is also placed in the hot plate for a postbake phase at $115^{\circ} \mathrm{C}$ for 100 seconds for obtaining the final chemical stabilization and substrate adhesion improvement. In order to obtain the lens profile, a reflow technique is used. The substrate with the $\mathrm{PR}$ array is placed at $180^{\circ} \mathrm{C}$ during 10 hours.

\section{B. Characterization}

In Figure 5, two photographs show the PR array before and after the reflow step, respectively, on the left, top view and on the right, tilted view.

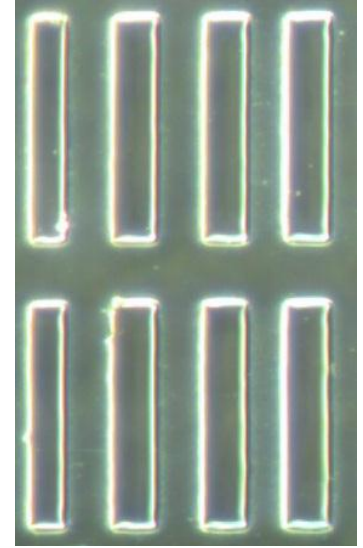

Figure 5: (a)

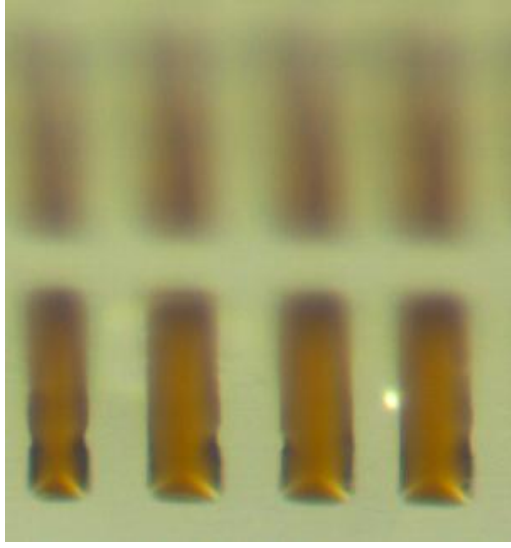

Figure 5: (b)
In Figures 6 (a) and (b) it is possible to see the profiles of the PR before and after the reflow process measured with a Veeco Dektak 150 profilometer.

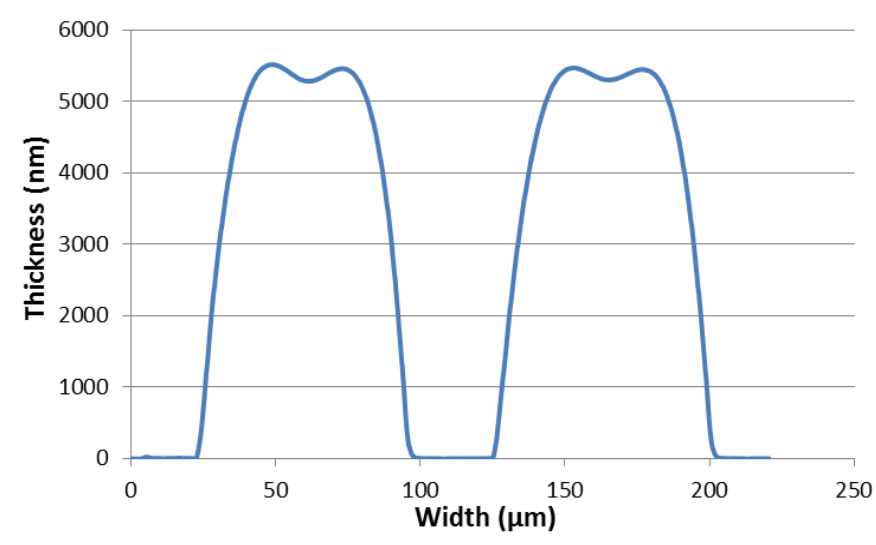

Figure 6: (a) - Profile of the PR array before the thermal reflow.

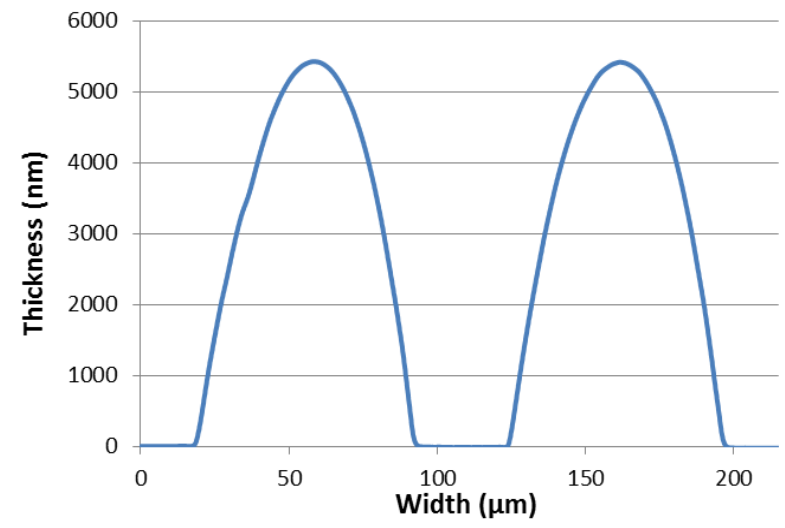

Figure 6: (b) Profile of the microlenses obtained after the thermal reflow step. 
This figure presents the typical initial profile seen during the fabrication of microlenses using PR [8,9]. After applying a temperature higher than the glass transition temperature of the $\mathrm{PR}$, the viscosity decreases and the consequent flow due to surface tension occurs. These structures are the first prototypes built using photolithography but the final size of the microlenses can be further reduced using a chromium-on-glass mask. Nevertheless, it is clear from the previous figures that the thermal reflow process permitted obtaining the desired microlens profile to concentrate the light into specific directions.

\section{CONCLUSIONS}

This paper presented a microlenses fabrication process for integration on a stereoscopic image sensor in CMOS technology for use in biomedical devices. The microlenses design started with the FEM simulations to set some parameters needed to fulfill the desired objectives. These objectives included the microlenses' $\mathrm{W} / \mathrm{L}$ dimensional ratio of 4.8 and 2.4 because of the size and pitch of the photodetectors. Each photodetector measures a specific wavelength with is difracted by the microlenses. The complete fabrication process was explained and the initial and final structures obtained were physically characterized. It was shown that the reflow step is what determines the actual microlenses profile. The several steps that comprise the photolithographic fabrication process were done for a first prototype but smaller ones are already being developed and tested using a chromium-on-glass mask and a mask aligner as the UV light source. The integration of such stereoscopic imaging sensor in a biodevice such as an endoscopic capsule for non-invasive examination of the digestive system is a very appealing solution for the presented fabrication technology.

\section{ACKNOWLEDGEMENTS}

This work was fully supported by the Portuguese Foundation for Science and Technology under the project FCT/PTDC/EEA-ELC/109936/2009.

\section{REFERENCES}

[1] I. Andorko, et al., "Hardware implementation of a real-time 3D video acquisition system", in proc. of 12th International Conference on Optimization of Electrical and Electronic Equipment (OPTIM 2010), Brasov, Ronaia, 20-22 May, pp. 920-925.

[2] S. Zeki, "The neurology of ambiguity", Consciousness and Cognition, Vol. 13, pp. 173-196, 2004.

[3] W. Qureshi, "Current and future applications of the capsule camera", Nature Reviews Drug Discovery, Vol. 3, pp. 447-480, May 2004.

[4] G. Iddan, et al, "Wireless capsule endoscopy", Nature, Vol. 405, pg. 417, May 2000.

[5] C. K. Chan, et al, "High-performance lithium battery anodes using silicon nanowires", Nature, Vol. 3, pp. 31-35, January 2008.

[6] M. Armand, M. Tarascon, "Building better batteries", Nature, Vol. 451, pp. 652-657, February 2008.

[7] J-C. Roulet, et al, "Fabrication of Multilayer Systems Combining Microfluidic and Microoptical Elements for Fluorescence Detection”, Journal of Microelectromechanical systems, Vol. 10, No. 4, December 2001 .

[8] A Emadi, et al, "Vertically tapered layers for optical applications fabricated using resist reflow", J. Micromech. Microeng., 19, 2009.

[9] F. O'Neill, et al, "Photoresist reflow method of microlens production Part I: Background and experiments", Optik 113, No. 9, pp. 391-404, 2002 